Pak. J. Agri., Agril. Engg., Vet. Sci., 2021, 37 (1): 42-48

ISSN: 1023-1072 (Print), ISSN: 2663-7863 (Online)

https://doi.org/10.47432/2020.37.1.6

\title{
INFLUENCE OF DIFFERENT COLOURS OF LIGHTS ON DEVELOPMENTAL STAGES OF RED FLOUR BEETLE (TRIBOLIUM CASTANEUM) AND ADULTS PHOTOTAXIS BEHAVIOR
}

\author{
N. Ahmed ${ }^{1}$, A. Ali ${ }^{*}$, S. A. Memon ${ }^{1}$, T. K. Qambrani ${ }^{2}$ and G. Khaliq ${ }^{3}$ \\ ${ }^{1}$ Department of Entomology, ${ }^{2}$ Department of Plant Pathology, ${ }^{3}$ Department of Horticulture, \\ Lasbela University of Agriculture, Water and Marine Sciences, Uthal, Balochistan, Pakistan
}

\begin{abstract}
Red flour beetle (Tribolium castaneum) is the major insect pest of store grain, and its single larvae can attack 88 grains during its life which leads to a considerable loss of quality and viability of grain. Therefore, to manage this insect pest present research was designed to check the effects of different colours of lights on the developmental stages of red flour beetle and their attraction on lights. Six (White, Yellow, Red, Green, Blue and Black) colours of light were used in the present experiment at $30 \pm 2{ }^{\circ} \mathrm{C}$ and $60 \% \pm 10 \%$ relative humidity $(\mathrm{RH})$. Results revealed that a longer incubation period $(4.17 \pm 0.18$, less hatching $(78.66 \pm 0.50 \%)$ and highest mortality $(21.34 \pm 1.11 \%)$ of $T$. castaneum eggs were observed on blue light, while a short incubation period $(3.03 \pm 0.51)$, highest hatching $(89.99 \pm 1.92 \%)$ and lowest mortality $(10.01 \pm 0.22 \%)$ were observed on white colour of light. However, a longer duration $(32.01 \pm 0.15,12.33 \pm 0.31)$ and maximum $(28.10 \pm 2.50,14.66 \pm 0.52)$ mortality of $T$. castaneum was found in larval and pupae stages on blue colour of light, while a short duration $(21.27 \pm 0.27,7.33 \pm 0.13)$ and minimum $(8.30 \pm 2.5,8.00 \pm 0.65)$ larval and pupal mortality (\%) were seen by red and yellow colour of lights. Similarly, an adult's longevity significantly reduced (48.33 \pm 1.76$)$ when beetles were exposed on blue light, whereas a significant (62.33 \pm 1.66$)$ increase in adult longevity was found on white colour of light. A highest $(33.33 \pm 0.56)$ attraction of red flour beetle was found on red colour of light, whereas, a lowest attraction was observed on blue (6.00 \pm 0.85$)$ and black $(10.00 \pm 1.55)$ colours of lights.
\end{abstract}

Keywords: developmental period, hatching percentage, light attraction, mortality, Tribolium castaneum

\section{INTRODUCTION}

Stored grains are broken by numerous store grain insect pests such as mites, weevil, beetles and moths, resulting in quantitative and qualitative losses (Rajendran and sriranjini, 2008). Store grain insect pests contribute to contamination in grains commodities through the occurrence of insect body fragments, excretions and dead insects. Red flour beetle (Tribolium castaneum) is the major insect pest of store grain that causes both quantitative and qualitative losses which spoil the flour by feeding, and it is a very common pest in flour mills, warehouses and grocery stores (Garcia et al., 2005). It is most economically key pest and has worldwide distribution (Padin et al., 2002). A single larva of $T$. castaneum can attack 88 grains during its life which leads to a considerable loss of quality and viability of grain (Atanasov, 1978). Karunakaran et al. (2004) examined that infestation reason by red flour

"Corresponding author: arifalirao@gmail.com beetle in unprotected kernel stored for nine months reduced germination virtually completely and augmented visually broken kernels from 9 to $39 \%$. Therefore, controlling the $T$. castaneum relies on the use of fumigation and conventional insecticides, such as chlorfluazuron and methyl bromide (Kim and Lee, 2013). Use of fumigation and chemical to secure stored product commodities by insect infestation and contamination while their continuously uses leads to problems of unpleasant residues and developed resistance in certain insect species (Huang and Subramanyam 2004; Khattak and Malik 1979); Salam and Shakoori 1990). Untill now, fumigation is the most effective method to manage these pests, but still has serious limitations, Such as fumigants do not penetrate some commodities in sufficient concentration to control some pests. However, in few instances, chemical treatments may have lethal influence on the grains or may leave adverse residues. Therefore, there is need to search for another pest management tactic for $T$. castaneum. 
Non-chemical control practices are attractive since they neither leave chemical residues in the grains and commodity nor do they cause resistance in insect pests (Padin et al., 2002). Amongst the non-chemical coordination, lights effects have been used to manage many insects pests of stored grains such as the Indian meal moth and cigarette beetle population (Sambaraju and Phillips, 2008). However, dark light and light of incandescent bulbs have short electric effectiveness and wide wavelengths; therefore, more efficient light source such as light emitting diodes (LEDs) have been used to control insects through light traps (Cohnstaedt et al., 2008; Lee et al. 2008). LEDs light traps have been broadly used to the management of Liriomyza trifolii and Trialeurodes vaporariorum insect pest (Kim and Lee, 2013). LEDs are most efficient because of their small size, light weight, low electric consumption, adjustable light intensity, long life time and capability to select specific wavelength (Tamulaitis et al., 2005); Yeh and Chung, 2009). Hassan and Khan (1998) reported that light can be used against stored product pests by the direct treatment of the commodities that provide a residue-free process of pest control, because lights do not cause residues in grains and also no significantly change the quality of the stored food material or stored kernels. Keeping in view, the store grain product of Pakistan is under threat due to red flour beetle infestations; present study was carried out to determine the effect of different colour of lights on life cycle parameters and adults phototactic behavior of red flour beetle in vitro.

\section{MATERIALS AND METHODS Culture of Red flour beetle}

The experiment was conducted in the Department of Entomology, Lasbela University of Agriculture, Water and Marine Sciences, (LUAWMS), Uthal, Balochistan. Red flour beetles (Tribolium castaneum) were collected from Uthal godowns and they were cultured in plastic boxes on wheat grain flour under laboratory conditions at $30 \pm 2^{\circ} \mathrm{C}$ and $60 \% \pm 10 \%$ $\mathrm{RH}$. The light source was purchased from science, international laboratory in Karachi. Six (White, Yellow, Red, Green, Blue and Black) different colour of lights were used in present study. Lights were used in the tope center of cardboard box with the size of $(30 \mathrm{~W} \times 30 \mathrm{~L} \times$ $30 \mathrm{H}$ ). During experiment it covered with black cloth. Each light was separately used in each cardboard box.
Plastic boxes were used for insects rearing and covered with cotton yarn. Wheat flour was provided as a diet in whole experiment. All experiments were replicated three times. Three hundred fresh eggs were collected and exposed on different light colours in petri dishes $(7 \mathrm{~cm}$ diameter) to check the hatching (\%) and their incubation periods. After hatching, 200 young larvae were collected and released on different light colours in plastic boxes $(3 \times 3 \times 4 \mathrm{~cm})$ covered with cotton yarn to observe the mortality (\%) and larval developmental period. One hundred, one day old pupae were collected and exposed to different light colours in petri dishes to find out the adult emergence (\%), and pupal days. After emergence 50 adults were collected and exposed to different lights to observe the adult longevity until the death of adults. The phototactic behavior of adults $T$. castaneum was also investigated. Transparent plastic pipe was used to find the phototactic behavior of adults $T$. castaneum. Five feet transparent plastic pipe was crossed in the middle of 6 light cardboard boxes. Transparent plastic pipe was banned at its one side with cotton net and closed with rubber and other hole was left open for insect entrance. Hundred adults of $T$. castaneum were collected randomly and exposed to different colour of lights to observe the adults phototactic behavior of $T$. castaneum. The light attraction data were recorded after 24 hours.

\section{Statistical analysis}

Data was analyzed with the help of SPSS (SPSS Inc., Chicago, IL, US) software and One Way Analysis of Variance (ANOVA) was used for data analysis and means were compared with Tukey test at $P<0.05$.

\section{RESULTS}

A significant $(P<0.05)$ hatching $(\%)$ of $T$. castaneum eggs was observed on white colour of light followed by yellow, red, green and black colour of light. While a minimum hatching percentage was observed on blue colour of light. No significant difference was observed among white, yellow, red, green and black colour of light except blue light (Figure 1).

A significant increased $(P<0.05)$ in mortality $(\%)$ of $T$. castaneum eggs was found on blue colour of light, however a significant decreased percentage was noticed on white colour of light, although no significant different were seen among white, yellow, red, green and black colour light (Figure 2). 
A maximum incubation period of $T$. castaneum eggs was recorded in blue colour followed by red, green, black and yellow colour light. However, minimum incubation period of eggs was observed in white color. Only significant $(P<0.05)$ difference was noticed on blue colour light as contrasted with other colours of lights. No differences were seen by white, yellow, red, green and black light as mentioned in Figure 3.

A longest larval developmental duration of $T$. castaneum was recorded on blue colour followed by black, green, white and yellow colour. While a minimum larval development duration was observed on red colour. Statistically, a significant $(P<0.05)$ difference was noticed on blue, black and green light as compared with white, yellow and red colours (Figure 4).

Larval mortality (\%) of $T$. castaneum significantly $(P<0.05)$ differed when larvae were exposed on blue and black colour of lights as compared to other colours of lights. while no significant changes were recorded in larval mortality on white, yellow, red and green colour of lights (Figure 5).

A longer duration of $T$. castaneum pupal period was recorded on blue colour of light, whereas a short duration of pupal period was observed on yellow colour of light. Only significant $(P<0.05)$ difference was found on blue colour of light as compared with others light colours in pupal period of $T$. castaneum, however, no difference was observed in pupal period in white, yellow, red, green and black colour of lights (Figure 6).

A mortality (\%) at pupal stage of $T$. castaneum was increased $(P<0.05)$ in pupae exposed to blue colour of light as compared to other colours, however a significant decreased of $T$. castaneum mortality (\%) at pupal stage was noticed on red colour of lights. But no difference was observed among white, yellow, red, green and black colour of lights (Figure 7).

A significantly $(P<0.05)$ increased adults longevity of $T$. castaneum was observed on white, yellow, red and green colour of lights, however a significant decrease adult longevity was observed on blue and black colour of lights. But no significant variation was observed among white, yellow, green and red colours of lights on adult's longevity of $T$. castaneum (Figure 8).

$A$ red colour of light was found more attractive to A highest significant $(P<0.05)$ lights attraction on adults stage of $T$. castaneum were found on red colour, while a lowest light attraction was observed on blue and black colour. Although no difference was seen among white, yellow and green colour for the attraction of $T$. castaneum adults (Figure 9 ).

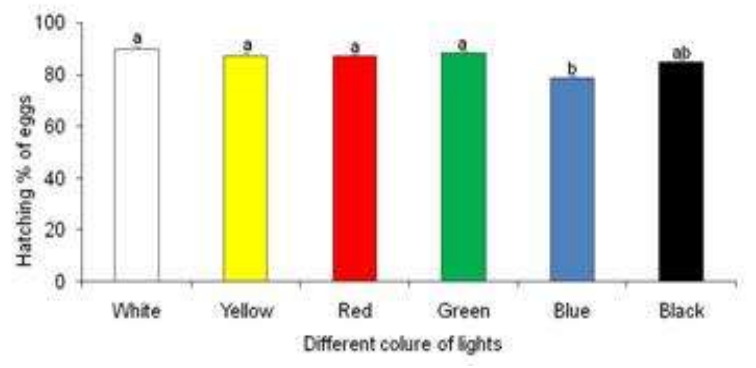

Figure 1. Influence of different colours of lights on the hatch ing (\%) of $T$. castaneum eggs. A and B indicating the differences $(P<0.05)$ among the groups

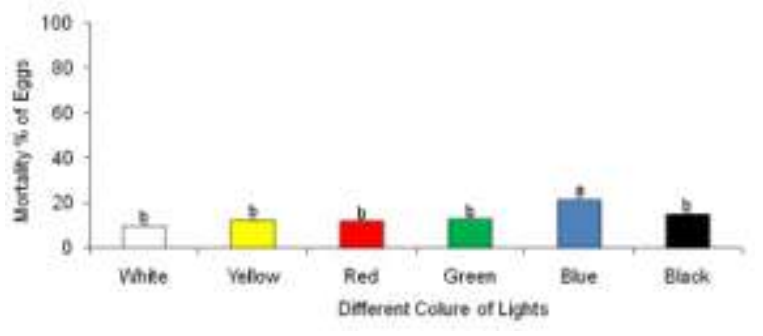

Figure 2. Influence of different colours of lights on the mortality (\%) of $T$. castaneum eggs. A and B indicating the differences $(P<0.05)$ among the groups

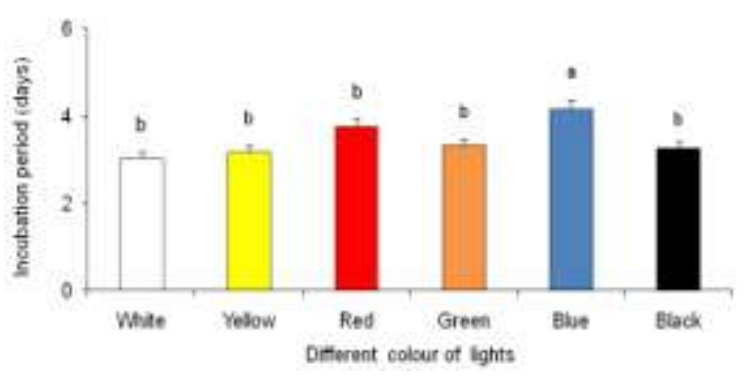

Figure 3. Influence of different colours of lights on the incubation period of $T$. castaneum eggs. A and B indicating the differences $(P<0.05)$ among the groups

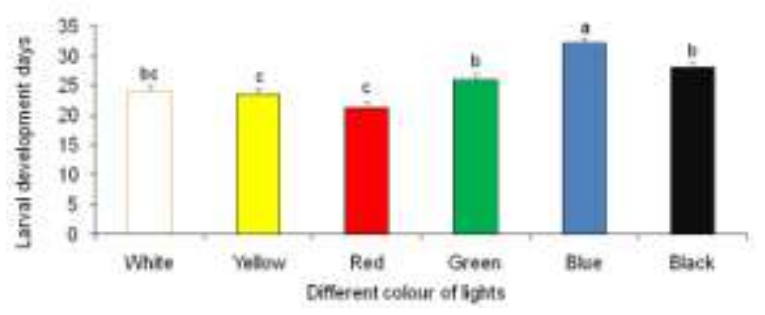

Figure 4. Influence of different colours of lights on the larval developmental days of $T$. castaneum. A, B and C indicating the differences $(P<0.05)$ among the groups 


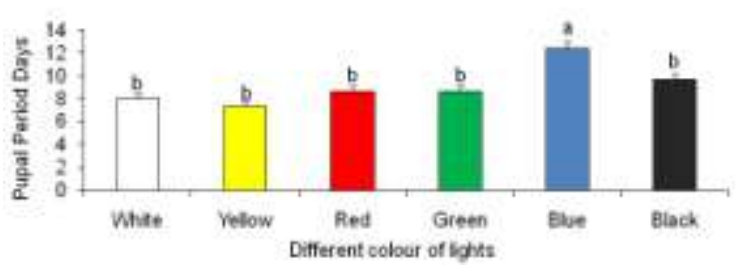

Figure 5. Influence of different colours of lights on the larval mortality (\%) of $T$. castaneum. A, B and $\mathrm{C}$ indicating the differences $(P<0.05)$ among the groups

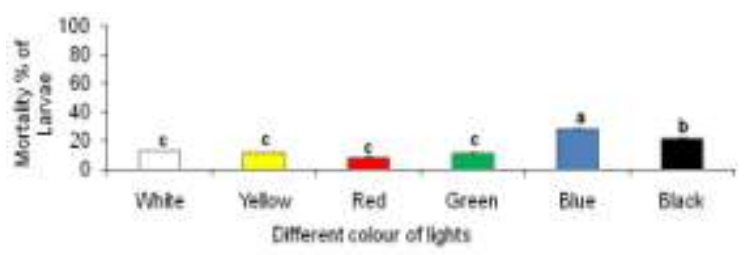

Figure 6. Influence of different colours of lights on the pupal developmental days of $T$. castaneum. A and B indicating the differences $(P<0.05)$ among the groups

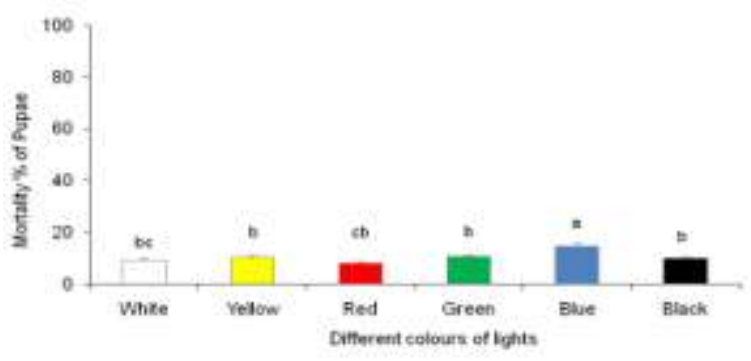

Figure 7. Different colure lights influence on the mortality (\%) of $T$. castaneum pupae.. . A, B and $\mathrm{C}$ indicating the differences $(P<0.05)$ among the groups

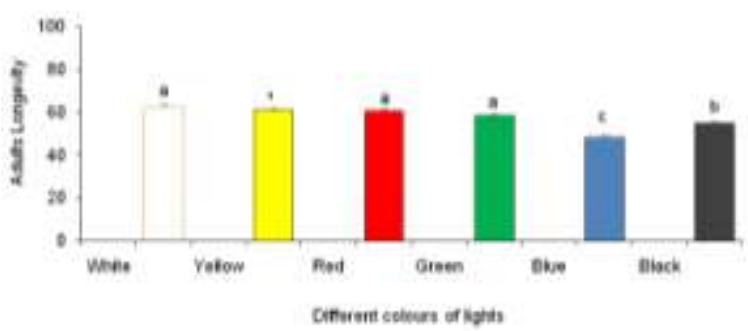

Figure 8. Influence of different colours of lights on the adults longevity of $T$. castaneum. . A, B and C indicating the differences $(P<0.05)$ among the groups

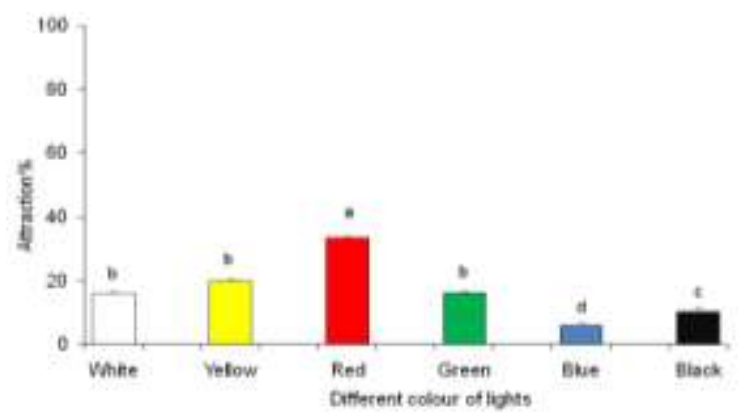

Figure 9. Adults Attraction of $T$. castaneum on different colure of lights. . A, B, C and D indicating the differences $(P<0.05)$ among the groups

\section{DISCUSSION}

Various colour of lights have significantly effect on orientation, locomotion, feeding, mating, ovipositor, adult emergence, and the development of insects (Khan et al., 1998). In present study we examined the influence of different colour of light on life history parameters and photo tactic behavior of red flour beetle in vitro and we observed that the less hatching $(78.66 \pm 0.50 \%)$ and longer $(4.17 \pm 0.18)$, incubation periods of eggs were found on blue light colour as compared with white, yellow, green, red and black colour lights. These Findings indicated that growth of embryo might be inhibited by the energy of blue light. A similarly results also were reported by Hori et al. (2014); Hori and Suzuki, (2017); Shibuya et al. (2018). They reported that blue light has negative impact on the eggs of $D$. melanogaste and Galerucella grisescens. However a highest hatching (\%) of eggs and lowest incubation period was recorded on white light. Agreed with the finding of Wang et al. (2013) who examined the highest eggs fecundity and fertility of bug Orius sauteri on white light.

Wang et al. (2013); Tariq et al. (2017) reported that blue light decrease the speed of metamorphosis of Orius sauteri and $B$. dorsalis and interruption in the growth of larvae to reach them at adult stage. Zhang et al. (2011); Ali et al. (2016) reported that black light enhance the larval period of $H$. armigera and $M$. separate. Similar results also were found in present study, and we observed longer $(32.01 \pm 0.15,28.03$ \pm 0.57 ), period of red flour beetle larvae on blue and black lights. While a short larval days $(21.27 \pm 0.27)$, and minimum mortality (8.30 \pm $2.5 \%$ ) of red flour beetle was observed on red light, finding of red flour beetle on red lights regarding short periods of larvae and mortality supporting with the results of Sheribha et al. (2010), who also found the less mortality and decline in the average larvae periods of $T$. castaneum on red light. The accurate mechanism of reaction to colour lights was not known, however it was assumed that different lights of colour produced harmful effects on the metabolism of grubs. Such metabolic changes have been observed by Narayan et al. (1959); Vaidya et al. (1974); Khan et al. (1998). Using of different colour light traps methods incorporating colored lights is also promising mechanism in suppressing the pest population of red flour beetle in any infested stored grains godowns.

A maximum pupal day $(12.33 \pm 0.31)$ was noticed on blue light, whereas a minimum pupal 
day $(7.33 \pm 0.13)$, was found on yellow light. Present results are supporting with the findings of Wang et al. (2013) who found that Orius sauteri took long time to reach at adult stage on blue light and less time were seen on green, yellow and white light for adults emergence. A highest mortality $(14.66 \pm 0.52 \%)$, at pupal stage was observed on blue light, however a lowest mortality $(8.00 \pm 0.65 \%)$, at pupal stage were observed on red light. similar consequence also were observed by Hori et al. (2014) because they has been observed the highest mortality of $D$. melanogaster, $C$. pipiens molestus and $T$. confusum at pupal stages on blue lights. However, the lowest mortality (\%) at pupal stage of $T$. castaneumon red light are supporting with the findings of Sheribha et al. (2010) who found the maximum adults emergence percentage of $T$. confusum on red lights.

In present results regarding adult's longevity of $T$. castaneum a significant decrease adult's longevity of red flour beetle was noticed when it's exposed on blue light and black light as compared with other lights. Present findings are agreed with the findings of Sheeba et al. (2000), theyreported that lights reduce the adult's life span in $D$. melanogaster and also agree with the investigation of Zhang et al. (2011); Ali et al. (2016) exposed that black light decrease the adult's longevity and reduce the fecundity of $\mathrm{H}$. armigera and $M$. separate. Further our results are similar with the results of Hori et al. (2014); Hori and Suzuki (2017), who reported that blue light, has negative effect on different developmental stages of Drosophila melanogaster and declined their adult's life span. Specific species and developmental stage specific photoreceptive part might be connected with the dangerous impact of blue and black light. Reactive oxygen species (ROS) might contribute to spoil caused by blue and black light. Therefore, the insect body is injured by ROS, which causes the reduction of adult's longevity. These results are indicating that blue light and black light could suppress the next generation of $T$. castaneum.

Red colour light was found more attractive to the adults of red flour beetles as compared with others lights. Previously, it was reported that blue light $(84.3 \%)$ was the most attractive to Sitophilus oryzae (L.), the rice weevil, followed by green, and UV LEDs lights (Jeon et al., 2012). However, green and the blue LEDs 1.3 and 1.5 times more attractive than the others lights. This consequence is change from our findings, where blue colour was undesirable for
$T$. castaneum. These changes may be due to the insect's species and $T$. castaneum depend on the luminance specific wavelength and light exposure time. Semeao et al. (2011) found that factors such as light intensity and contrast with colour background may be impact the level of adults captured. Peitsch et al. (1992) Briscoe and Chittka (2001) investigated that Insects typically have photoreceptor that react to ultraviolet, blue and green lights. Furthermore, Bees of several species have natural preference for UV-blue and blue flowers (Giurfa et al., 1995); Raine et al., 2006). A number of insects have blue, green and UV photoreceptor in their eyes (Brisco and chittka, 2001). Vaishampayan et al. (1975) reported a phototactic response of Trialeurodes vaporariorum (westwood) to yellow, green and UV illuminance. Photoreceptor of $T$. castaneum adults has a single long wavelength opsin-across the entire retina (Jackowska et al., 2007). Adults showed highest attraction on red light may be the reason of long wavelengthopsin of retina. On the basis of our findings, it was suggested that, 24 hours exposure of red light to $T$. castaneum was the most suitable in attracting $T$. castaneum adults for protecting stored foods/grains.

\section{CONCLUSION}

The present results revealed that different colours of lights have ability to disturb the physiological activities of T.castaneum. Colours may be considerable aspects of integrated pest managements alongside the other insects control application. Presents findings are essential towards providing baseline information for established pest control technique using various colours of lights. Useful pest control could be realized by clarifying the most lethal colour of lights of the targeted developmental stages such as eggs and pupae could be targeted due to unmovable stages. Larvae and adult insects that live in limited space could also be targeted, such as sanitary insect pest or stored grain insect's pest. Use of different colour lights for pest control, we must first indentify the effective colour of each developmental stages for each targeted species. Furthermore, this study will provide important information on insect photobiology for researchers and extension workers.

\section{ACKNOWLEDGEMENT}

We acknowledge this research work to all Departmental staff of Entomology, Lasbela University of Agriculture, Water and Marine 
Science (LUAWMS), Uthal, Balochistan for their support during my research work.

\section{AUTHOR'S CONTRIBUTION}

N. Ahmed: Research conducted

A. Ali: Experimental designed

S. A. Memon: Paraphrasing

T. K. Qambrani: Paraphrasing

G. Khaliq: Manuscript reviewed

\section{REFERENCES}

Ali, A., M. A. Rashid, Q. Y. Huang and C. L. Lei. 2016. Effect of UV-A radiation as an environmental stress on the development, longevity, and reproduction of the oriental armyworm, Mythimna separata (Lepidoptera: Noctuidae). Environmental Science and Pollution Research, 23 (17): 17002-17007.

Atanasov, K. H. 1978. Damage by the rust red grain beetle to stored grain and its products. Rastitelna Zashchita, 26: 19-20.

Briscoe, A. D. and L. Chittka. 2001. The evolution of color vision in insects. Annual Review of Entomology, 46 (1): 471-510.

Cohnstaedt, L. E. E, J. L. Gillen and L. E. Munstermann. 2008. Light-emitting diode technology improves insect trapping. Journal of the American Mosquito Control Association, 24 (2): 331.

García, M., O. J. Donadel, C. E. Ardanaz, C. E. Tonn and M. E. Sosa. 2005. Toxic and repellent effects of Baccharis salicifolia essential oil on Tribolium castaneum. Pest Management Science: Formerly Pesticide Science, 61 (6): 612-618.

Giurfa, M, J. Nunez, L. Chittka and R. Menzel. 1995. Colour preferences of flower-naive honey bees. Journal of Comparative Physiology, 177 (3): 247-259.

Hasan, M., M. S. Jahan and A. R. Khan. 1998. Effect of UV radiation on the uzi-fly, Exorista sorbillans Wiedemann, an end parasitoid of the silkworm, Bombyx mori L. International Journal of Tropical Insect Science, 18 (1): 87-91.

Hasan, M. and A. R. Khan. A. 1998. Control of stored-product pests by irradiation. Integrated Pest Management Reviews, 3 (1): 15-29

Hori, M., K. Shibuya, M. Sato and Y. Saito. 2014. Lethal effects of short-wavelength visible light on insects. Scientific Reports, 4: 1-6.

Hori, M. and A. Suzuki. 2017. Lethal effect of blue light on strawberry leaf beetle,
Galerucella grisescens (Coleoptera: Chrysomelidae). Scientific Reports, 7 (1): 1-6.

Huang, F., B. Subramanyam and M. D. Toews. 2004. Susceptibility of laboratory and field strains of four stored-product insect species to spinosad. Journal of Economic Entomology, 97 (6): 2154-2159.

Jackowska, M., R. Bao, Z. Liu, E. C. McDonald, T. A. Cook and M. Friedrich. 2007. Genomic and gene regulatory signatures of cryptozoic adaptation: Loss of blue sensitive photoreceptors through expansion of long wavelength-opsin expression in the red flour beetle Tribolium castaneum. Frontiers in zoology, 4 (1): 1-11.

Jeon, J. H., M. S. Oh, K. S. Cho and H. S. Lee. 2012. Phototactic response of the rice weevil, Sitophilus oryzae Linnaeus (Coleoptera: Curculionidae), to light-emitting diodes. Journal of the Korean Society for Applied Biological Chemistry, 55 (1): 35-39.

Karunakaran, C., D. Jayas and N. D. G. White. 2004. Identification of wheat kernels damaged by the red flour beetle using $X$-ray images. Biosystems Engineering, 87 (3): 267-274.

Khattak, S. U. K. and M. M. Malik. 1979. Reproductive potential of red flour beetle, Tribolium castaneum Herbst, adults after exposure to gamma radiation and vacuum. Pakistan Journal of Zoology, 11: 173-176.

Kim, M. G., J. Y. Yang and H. S. Lee. 2013. Phototactic behavior: repellent effects of cigarette beetle, Lasioderma serricorne (Coleoptera: Anobiidae), to light-emitting diodes. Journal of the Korean Society for Applied Biological Chemistry, 56 (3): 331333.

Lee, K. P., S. J. Simpson and K. Wilson. 2008. Dietary protein quality Influences melanization and immune function in an insect. Functional Ecology, 22: 1052-1061.

Narayan, E. S., R. Lal, G. W. Rahlkar, G. R. Sethi and P. N. Saxena. 1959. Studies on the effects of beta radiations on insects. The effects of beta radiation (irrigation of egg stage and first instar larvae) on the life history of Corcyra cephalonica Stainton. Proceeding of the Indian Academy of Sciences-Section B, Springer India, 50 (2): 82-87.

Padín, S., G. Dal Bello and M. Fabrizio. 2002. Grain loss caused by Tribolium castaneum, Sitophilus oryzae and Acanthoscelides 
obtectus in stored durum wheat and beans treated with Beauveria bassiana. Journal of Stored Products Research, 38 (1): 69-74.

Papadopoulou, S. C. H. and C. T. H. Buchelos. 2002. Comparison of trapping efficacy for Lasioderma serricorne (F.) adults with electric, pheromone, food attractant and control-adhesive traps. Journal of Stored Products Research, 38: 375-383.

Peitsch, D., A. Fietz, H. Hertel, J. de Souza, D. F. Ventura and R. Menzel. 1992. The spectral input systems of hymenopteran insects and their receptor-based colour vision. Journal of Comparative Physiology A, 170 (1): 23-40.

Raine, N. E., T. Ings, C. Dornhaus, A. N. Saleh and L. Chittka. 2006. Adaptation, genetic drift, pleiotropy, and history in the evolution of bee foraging behavior. Advances in the Study of Behavior, 36: 305-354.

Rajendran, S. and V. Sriranjini. 2008. Plant products as fumigants for stored-product insect control. Journal of stored products Research, 44 (2): 126-135.

Saleem, M. A. and A. R. Shakoori. 1990. The toxicity of eight insecticides to sixth instar larvae and adult beetles of Tribolium castaneum (Herbst.). Pakistan Journal of Zoology, 22 (3): 207-216.

Sambaraju, K. R. and T. W. Phillips. 2008. Responses of adult Plodia interpunctella (Hubner) (Lepidoptera: Pyralidae) to light and combinations of attractants and light. Journal of insect behavior, 21 (5): 422-439.

Semeao, A. A., J. F. Campbell, R. J. Whitworth and P. E. Sloderbeck. 2011. Response of Tribolium castaneum and Tribolium confusum adults to vertical black shapes and its potential to improve trap capture. Journal of Stored Products Research, 47 (2): 88-94.

Sheeba, V., V. K. Sharma, K. Shubha, M. K. Chandrashekaran and A. Joshi. 2000. The effect of different light regimes on adult lifespan in Drosophila melanogaster is partly mediated through reproductive output. Journal of Biological Rhythms, 15 (5): 380-392.

Sheribha, P. R. B., A. P. Jinham, S. S. M. Das and K. R. Jasmine. 2010. Management of
Tribolium castaneum (Herbst) based on hue response. Turkish Journal of Zoology, 34 (3): 367-375.

Shibuya, K., S. Onodera and M. Hori. 2018. Toxic wavelength of blue light changes as insects grow. PloS one, 13 (6): e0199266.

Tamulaitis, G., P. Duchovskis, Z. Bliznikas, K. Breivë, K. Ulinskaitë, R. Brazaitytë, A. Novièkovas and A. Pukauskas. 2005. Highpower light-emitting diode based facility for plant cultivation. Journal of Physics D: Applied Physics, 38 (17): 3182-3187.

Tariq, K., M. Noor, M. Hori, A. Ali, A. Hussain, W. Peng and $H$. Zhang. 2017. Blue lightinduced immune suppression in Bactrocera dorsalis adults, as a carryover effect of larval exposure. Bulletin of Entomological Research, 107 (6): 734-741.

Vaidya, V. G., R. M. Kothari, N. N. Gedhole and S. P. Patil. 1974. Effect of gamma irradiation on the protein metabolism in Drosophilidae (Diptera). Radiation Research. 57 (3): 545-550.

Vaishampayan, S. M., G. P. Waldbauer and M. Kogan. 1975. Visual and olfactory responses in orientation to plants by the greenhouse whitefly, Trialeurodes vaporariorum (Homoptera: Aleyrodidae). Entomologia Experimentalis et Applicata, 18 (4): 412-422.

Wang, S., X. L. Tan, J. P. Michaud, F. Zhang and X. Guo. 2013. Light intensity and wave length influence development, reproduction and locomotor activity in the predatory flower bug Orius sauteri (Poppius) (Hemiptera: Anthocoridae). Bio-control, 58 (5): 667-674.

Yeh, N. and J. P. Chung. 2009. High-brightness LEDs-energy efficient lighting sources and their potential in indoor cultivation. Renewable and Sustainable Energy Reviews, 13 (8): 2175-2180.

Zhang, C. Y., J. Y. Meng, X. P. Wang, F. Zhu and C. L. Lei. 2011. Effects of UV-A exposures on longevity and reproduction in Helicoverpa armigera, and on the development of its $F_{1}$ generation. Insect Science, 18 (6): 697-702. 